\title{
Radios y ciudadanías, posibles construcciones desde las ciberculturas
}

\author{
Radios and citizenships, possible constructions from cybercultures
}

Santiago Rengifo Orozco ${ }^{1}$

\author{
Rengifo O, Santiago \\ miradas $\mathrm{N}^{\circ} 14-2016$. ISSN: 0122 994X Págs 53 - 60 \\ Recepción: Junio 18 de 2016 \\ Aprobación: Octubre 01 de 2016 \\ Publicación: Diciembre 20 de 2016
}

\section{Resumen}

La radio entendida como artefacto cultural que permite expresar y comprender realidades, también es un medio por el cual se reconocen ciudadanías. Tal vez es necesario pensar que la palabra y el sonido, potencian una suerte de conexión entre memoria y construcción de otras ciudades posibles. En este sentido, es necesario hablar de ciberciudadanías e indagar cómo las temporalidades inciden en la comprensión y posterior memoria que permita otras ciudadanías. Así mismo la cibercultura rompe con las formas en que las relaciones sociales se vienen dando, de esta manera es vital un reconocimiento holístico del nuevo ecosistema que ya ha empezado a configurarse.

Palabras clave: Temporalidad, artefacto cultural, ciudadanías, radio, cibercultura

\begin{abstract}
The radio, mostly understood as cultural artifact that allows people to express and understand reality, it is also a way in which citizenships are recognized, maybe is necessary to think that word and sound reinforce a sort of cohesion between memory and construction of other possible cities. In that sense, is necessary to speak about cities and investigate how temporality influence, in the understanding and its subsequent historical memory so it can lead to other cities. In addition to that, the cyberculture breaks with all the models, in a way that social relationships can grow. Is essential then, to give integral recognition to the new ecosystem that has already started to set up.
\end{abstract}

Key Words: temporality, cultural artifact, citizenships, radio, cyberculture.

1 Licenciado en Comunicación e Informática Educativa. Estudiante de Maestría en Comunicación Educatia. Docente catedrático de la Universidad Tecnológica de Pereira.srengifo@utp.edu.co 


\section{Introducción}

El rápido avance de las tecnologías no permite procesar la fuerza con que se están dando los cambios, dicho esto es vital empezar a señalar las otras formas en que internet y la virtualidad generan otros procesos; identificar en internet y la radio artefactos culturales que pueden potenciar ciudadanías, se ha vuelto una deuda latente para quienes creen que desde las ciberculturas se puede trabajar para empezar a potenciar otras realidades posibles, dice Pierre Levy que la virtualidad permite reconocer posibilidades que antes no se pensaban, de ésta manera habla de la interactividad como muchos escenarios de participación, dónde se procura por construir otras realidades: Levy (2007) "El término «interactividad» designa generalmente la participación activa del beneficiario de una transacción de información." Pág 43. Ésta apertura a la información abre otras perspectivas para viejos y nuevos medios pero además en la postura de lo que significa lo virtual, permite hablar de otras construcciones que son potencia del ser que se ve inmerso en nuevas realidades, la interacción juega un papel importante como punto de partida de dicha reflexión así como en la construcción de hiperrealidades y seguro que esa característica abarca un nuevo mundo por comprender, desde allí surge como inevitable revisar la inmersión y fusión de algunos medios como la radio, pero además y no menos importante lo que de ellos se espera o al menos están dispuestos a potenciar.

\section{¿Artefactos ciberculturales?}

Los artefactos culturales como construcciones de sentido han permeado las formas en que interactuamos, son herramientas tecnológicas del ser, un entramado ontológico que parece cada vez más instrumental. Sin embargo, se suele desechar la fuerza implícita que las recubre, tal vez es primordial reconocer que las tecnologías del hombre son creaciones de sentido, pero que siempre, han tenido una carga de inercia. Algunos artefactos se recubren de sensibilidad y le dan al hombre otro poder: el de la emancipación, la salida y la búsqueda de otros caminos.

¿Qué sucede cuando los medios o artefactos se usan para bienes comunes? algo especial sucede con internet y las relaciones que ha empezado a construir: miles de personas se han empezado a encontrar en la web $\mathrm{y}$ aún no potencian esta prótesis mental que es internet. Lo cierto es que siempre estaremos en constante revisión de lo que los medios nos permiten para volcarnos hacia una sociedad más justa. El primero de ellos, por nombrar sólo algunos, fue el lenguaje, hermosa primera forma de nombrarnos y que constituye una forma de explicar la ontología de un pueblo a través de la forma cultural mínima. Al respecto, dice Ong (1982): "Las palabras son sonidos. Tal vez se las "llame" a la memoria, se las "evoque". Pero no hay dónde buscar para "verlas". No tienen foco ni huella (una metáfora visual, que muestra la dependencia de la escritura), ni siquiera una trayectoria. Las palabras son acontecimientos, hechos" (pág. 38). La palabra como nuestra primera forma comunicativa, es una ventana abierta a un mundo de la imagen, seguro que de complejidades, es la cultura que nos media todo el tiempo y de allí, que la radio se inscriba con tanta fuerza en la recepción.

Volvamos entonces sobre la naturaleza del artefacto, de suerte que se conciba como una virtualidad e instrumental, pero en relación constante con el quehacer propio del hombre, se podría decir de una manera muy determinista que está presente en todo, lo cual nos lleva a indagar en la relación que tiene con el hombre; Benedict Anderson dice al respecto (1993): "Mi punto de partida es la afirmación de que la nacionalidad, o la "calidad de nación" 
-como podríamos preferir decirlo, en vista de las variadas significaciones de la primera palabra-, aI igual que el nacionalismo, son artefactos culturales" (pág. 18) Intenta Anderson plantear la idea de la "nación" como una creación que influye fuertemente en las sociedades, creaciones conceptuales que se convierten en motor de ideas; sin embargo conviene concebir el artefacto cultural como herramienta que vincula a las personas cumpliendo funciones sociales, pues bien el de los medios y en especial el de la radio ha trascendido fuertemente como fórmula emancipadora aún a la espera de demostrar dichas posibilidades, al respecto de ésta perspectiva de la radio como artefacto aporta Bertolt Brecht: Brecht "Recuerdo una vieja historia en la que se quería demostrar a un chino la superioridad de la cultura occidental. El chino preguntó:"¿Qué tenéis?” Le dijeron: "Ferrocarriles, automóviles, teléfono." "Siento tener que decirles replicó el chino cortésmente - que esto nosotros ya lo hemos vuelto a olvidar." Por lo que a la radio respecta, tuve en seguida la terrible impresión de que era un aparato incalculablemente viejo, que quedó relegado en el olvido por el Diluvio Universal." Queda claro entonces que la radio es un elemento cutural de larga duración en el tiempo y que no está supeditado a la técnica, la descubierta por Marconi, en cambio lo que recrea Brecht acá es la creación de un artefacto, una tecnología del hombre que permitió el desarrollo cultural, la palabra o la radio como el nombre que damos hoy al medio, es solo un nombre que damos pero que está exento de sentido, es de ésta manera como aparentemente se vuelve una simple tenología, nuevamente Levy repasa sobre éste tema: Levy (2007): “ ¿Es la tecnología un actor autónomo, separado de la sociedad $y$ de la cultura., una entidad pasiva $y$ percutida por un agente exterior? pág 6 parece pues que la tecnología adquiere tintes futurísticos y sobrenaturales, haciendo parte de pensamientos o ideales deidisticos para inventos que lo que han sido es extensiones de nuestro cuerpo y mente, pero continúa levy: "Sostengo por el contrario que la técnica es un ángulo del análisis de los sistemas sociotécnicos globales, un punto de vista que pone el acento sobre la parte material y artificial de los fenómenos humanos, y no una enticlad real, que pudiera existir independientemente del resto, que tuviera efectos distintos y actuara por sí misma" y clarifica levy con ésta apreciación su postura sobre la técnica y la tecnología como mediadores en el desarrollo social y cultural de las sociedades, es allí donde es permitido decir que la tecnologpia supera la máquina y se convierte en esa potencia del ser creador.

¿Es entonces, un artefacto cultural, una creación que permite incluir a los nuevos medios? decíamos en palabras anteriores que se comporta como una virtualidad humana $\mathrm{y}$, hay que ver, cómo lo virtual ha empezado a transformar nuestras formas sociales; aunque en el pasado no permitiéramos lo virtual como una categoría "real", hoy es posible retener éste concepto para referirnos a casi todo lo que sucede en la web, y en este sentido, capta especial atención la relación que existe entre radio e internet, pues las ciberculturas han encontrado allí un camino importante, ya que como artefacto cultural, se dispone a recibir las relaciones culturales de la web, es bien sabido que los medios cambian cuando aplican su convergencia en internet, y la radio no ha sido ajena a esto, algunos hablan de ciberradios para referirse a una de las muchas formas en que se producen contenidos radiofónicos; sin embargo, conviene revisar perimero la definición que hiciera Pierre Levy sobre ciberculturas: Levy (2007) "En cuanto al neologismo "cibercultura», designa aquí el conjunto de las técnicas (materiales e intelectuales), de las prácticas, de las actitudes. de los 
modos de pensamiento y de los valores que se desarrollan conjuntamente en el crecimiento del ciberespacio" (pág. 1). Es entonces la cibercultura una invención virtual, un artefacto cultural, una creación humana usada para un bien común, y como tal, está estrechamente relacionada con la radio, pues el sonido y la palabra siguen haciendo parte de nuestras relaciones $\mathrm{y}$ construcciones.

\section{Ciudadanías posibles}

Todos podemos definir con exactitud lo que es una ciudad, o al menos lo que nuestra realidad nos permite describir de la misma, podría decirse, de nuevo involucrando a Benedict Anderson y su concepto de artefactos culturales, que la ciudad, ya por fuera del territorio, de la estructura y la arquitectura, es un artefacto, construcción hiperreal, definida desde el colectivo como un espacio en el que todos confluimos, existimos y construimos una vida, dicho lugar adquiere significado cuando nos permitimos hacer parte de él de forma consciente y significativa, los griegos delimitaron la ciudad a dos conceptos, "asty" que significa el lugar en cuanto lo material y "polis" para referirse a la comunidad organizada, de lo anterior podemos decir que aunque sería válido relacionar la ciudad con lo virtual de Levy, también es cierto que se anteponen, Aristóteles decía sobre la ciudad:Aristóteles (2000) "El ciudadano no es ciudadano por residir [en un lugar determinado] " esto nos permite pensar que no hace falta pertenecer al lugar, es necesario ser partícipe, es por eso que se reconoce la potencia que implica la ciudadanía, de la acción se encargará el trasegar de lo que implica la cibercultura Levy (2007)'”En la aceptación filosófica, es virtual lo que no existe más que en potencia y no en acto"'(pág33) Surge una nueva forma de definir la ciudadanía, las ciberciudades, espacios que se construyen a partir de esa potencia virtual que va cambiando y recreando la forma de vivir la ciudadanía

La llegada de internet comenzó por configurar nuevos paradigmas y estructuras; a lo mejor el mito de la caverna se reinventa cada día como si el hombre necesitara estar en una búsqueda constante, reinventándose, inacabado, siempre esperando poder ser mejor; es allí donde surgen las ciberculturas para permitirle nuevas miradas $\mathrm{y}$ otras posibles formas de reconfigurarnos, ya no como una esperanza, tal vez como un salvavidas o como la perspectiva hacia un futuro prometedor.

La ciudad que se empieza a construir hoy en las virtualidades nos ha lanzado a la latente posibilidad de muchas otras formas, llenos de complejidad y conviviendo en un ecosistema sistémico, en las ciberculturas se posibiliten reencuentros con ciudadanías nuevas. Hoy es posible avizorar otras socialidades que se van construyendo a raiz de las nuevas mediaciones que se disparan Galindo (2005): "Los ciudadanos reales llegan al mundo virtual y lo colonizan demasiado rápido, y poco a poco descubren que pueden ser otros, que pueden ser muchos, que pueden ser lo que quieran, lo que desean, lo que temen, lo que inventan" (pág. 161). La figura de un nuevo ciudadano se presenta como punto de partida de otros posibles consumos, el trabajo, la economía y la producción, son elementos que empiezan a cambiar, y si en un momento partimos en críticas contra los mass media y su imposibilidad de generar cambios, la multimodalidad de internet abre una nueva discusión y es esencial entonces, comprender los posibles caminos implica pensar en otros espacios que se desenlazan en las ciberciudades: Galindo (2005) " $L a$ nueva ecología urbana es un tejido de ciudades interconectadas más allá de la tierra y el mar, de las fronteras con visas y pasaportes, una configuración de lo urbano que se configura por las conexiones de las 
líneas aéreas, por los canales de televisión vía satélite, por las ondas invisibles de los impulsos eléctricos que cruzan el aire por todo el mundo conocido. $Y$ aún faltaba más, llegó internet"'(pág 160)

Esta ardua travesía compromete nuevos medios y reivindica otros, antes recordábamos la palabra como tecnología, así mismo se reconoce su poder desde el diálogo, un medio que muchos dan por perdido, pero ¿no es más oral la tecnología en nuestra época?, la radio en la virtualidad permite encontrarse con nuevas formas desde lo sonoro y aún es más fuerte si se tiene en cuenta o se reconoce en el nuevo ciudadano una figura que rompe con las mediaciones y reconfigura los modos de comunicarnos reventándolos, allí se puede interpretar que el poder de las ciberciudadanías se traducee en muchas otras formas de estar en el mundo, emancipación que ya empieza a generar nuevas relaciones.

Ahora bien, comprender esas posibilidades nos ubica en la relación con los artefactos culturales y lo que ellos permiten para generar procesos conducentes a contribuir en la formación de nuevas ciudadanías que se configuran en una apuesta por otras formas posibles, donde son importante las relaciones que se imbrican así: Bernal ("Las redes se crean, se establecen y se desarrollan en el ciberespacio, en la vida virtual de los impulsos electrónicos. La creación de redes dentro del ciberespacio conformación de vínculos por medio de la participación continua por parte de los sujetos sociales, tanto para dar apoyo a acciones políticas inmediatas, como en foros y debates, el correo electrónico, se estructura una matriz con múltiples nodos de acceso y retorno nodal donde la comunicación se convierte en el vehículo simbólico y son la clave para la vida virtual de un nuevo espacio social, el ciberespacio” (pag 2) Bernal . Surge entonces la propuesta de hacer de dichas ciudadanías abiertas, que permitan volver al diálogo como punto de partida de todo proceso social, esos otros reconocimientos donde la radio empiece a jugar un papel importante en esas nuevas construcciones, y si el espacio es el de las ciberculturas el camino se abre a lo que llamaríamos el empoderamiento del medio, miles de usuarios están empezando a usar la radio en internet con diversos fines, la producción radiofónica es ahora la de los nuevos ciudadanos.

\section{Temporalidades, construcción de la realidad o la hiperrealidad}

Cuando hablamos de ciudadanías surge una pregunta por el como esta se involucra en las formas en que el hombre se desenvuelve, allí se puede advertir que tanto la radio como las ciberculturas cambian las formas en que percibimos el mundo, es por eso que el concepto de temporalidad permite revisar como se están constuyendo dichas realidades, éstas temporalidades indican de qué manera se teje la trama del presente, pasado y futuro, ésto funciona como simple percepción o construcción de la realidad, dicha conexión es cronológica de un estado del tiempo: (Heidegger)“El tiempo es algo en lo que sepuede fijar algo arbitrariamente un punto que es una ahora, de tal manera que en relación con dos puntos temporales siempre se puede decir que uno es anterior y otro posterior" pag 31

\section{Pero y ¿qué es la temporalidad?}

En primer lugar Heiddeger sugiere que comprender el tiempo es una tarea que se nos permitirá si nos familizarimos con el concepto de Dasein, respuesta ontológica de la filosofía alemana y que propone la existencia del ser en un ahora, el "ser ahí", Heidegger lo define como un ser que nunca está determinado o acabado, de ésta manera una de las apreciaciones más importantes es que ese "ser ahí" también es una vez que está con los otros "ser ahí", es decir 
en construcción con otros: "Heidegger (2003)"...mayormente y como término medio no soy yo mismo mi ser-ahí, si no que lo son los otros; yo soy con los otros, y los otros son igualmente con los otros" (pag 38) Todo esto supone que el ser se hace gracias a las temporalidades, eso implica la aparición de una cotidianidad, espacio donde se construye la temporalidad.

Lo temporal pertenece a lo cotidiano, donde se realiza el tiempo en un ahora, acontecimiento que se renueva, dicha anticipación del futuro del "ser ahí" es la realización temporal, porque somos seres distintos, a cada momento nos rehacemos e inevitablemente el tiempo nos lleva a la muerte, ahora bien, el cotidiano le construye al "ser ahí" temporalidades que le permiten reconocer un momento y no el otro, de allí que tengamos conciencia de tiempo, dicho sea de paso que esto se permite gracias a los otros, al menos en dicho imaginario cronológico Heidegger (2003)"El reloj que uno tiene, cualquier reloj, muestra el tiempo del ser-uno-con-los-otros-en-elmundo" (pág 52) sin embargo esos otros son ajenos a mi ser ahí, eso implica que constuyen otras temporalidades, otras realidades.

Ya sabemos que las temporalidades definen la forma en que los seres humanos comprenden la realidad, en la actualidad hablamos de hiperrealidades donde la construcción se convierte en una simulación, un algo virtual que carece de posesión, más bien es una cosificación prestada donde lo real se vuelve hecho de otros, esto se da gracias a dicha exposición de la cotidianidad con los otros "ser ahí" que nos permite comprender lo que es "real", ahora bien es posible pensar en que dicha realidad se convierte en una simulación de la misma, Baudrillard preferiría creer que la simulación hace parte de nuestra forma de ver la realidad: Baudrillard (1978) " $L a$ simulación no corresponde a un territorio, a una referencia, a una sustancia, sino que es la generación por los modelos de algo real sin origen ni realidad: lo hiperreal. (Pág. 5). Entonces es cada vez más claro que las temporalidades recrean realidades o simulaciones, pero no es tal el hecho de la simulación, Baudrillard intenta decirnos con esto que la realidad ya no es pura, sin embargo las hiperrealidades se configuran como la forma de comprender otros mundos posibles, la diversidad de contextos, otras temporalidades de otros "ser ahí", la cotidianidad con los otros y ahora con la virtualidad, de eso sabe muy bien la cibercultura que a cada instante nos sumerge en nuevas realidades.

Ahora bien, sabemos que el mundo, lo cotidiano se construye gracias a dichas temporalidades, además de las percepciones de la realidad entonces el "artefacto cultural" que medie nos rehace todo el tiempo, por eso al hablar de un "hecho" es complejo decir que lo percibimos fielmente, es más adecuado decir que se diluye en la relación que tenemos con él, un "acontecimiento" por el contrario dice Hartog: Hartog (2007) "Aquí, por el contrario, se percibe inmediatamente el acontecimiento, "según el orden recibido de la estructura, como idéntico al acontecimiento original". No llega, sino que regresa, no es único, sino repetición" (Pág. 56). La realidad se hace compleja a cada momento y la forma en que se puede construir realidades, esa temporalidad nos sigue midiendo el mundo, el espacio, ahora si ésta construcción la percibimos desde la radio, la palabra, la oralidad, por eso surge la pregunta que indaga por el recuerdo de lo real: ¿acaso es más potente el discurso oral que continúa circulando y se pierde para que perdure en la memoria o por el contrario es un proceso que permite aquella recuperación de la que tanto carece nuestra sociedad? de esta manera vale la pena analizar las palabras de José Luis Fernández: Fernández (2003) "Si el ejercicio básico de la memoria es situar un 
espacio en el tiempo, puede pensarse que también tendrán su importancia los modos específicos de procesar la temporalidad que ejerce cada medio. Debe tenerse en cuenta, entonces, que escuchar la radio implica estar situado complejamente en un entrecruzamiento espaciotemporal de lo discursivo con su contexto de recepción. Si bien se puede recibir situado frente al parlante, mientras se despliega temporalmente el texto radiofónico, esa posición no es la única posible.

Lo temporal nos decora nuevamente un entramado que define lo que percibimos, ésta mediación puede ser asincrónica, en un tiempo único y fiel a mis necesidades, en la virtualidad se permite que ese tiempo sea infinito y reproducible en muchas otras temporalidades, eso permite comprender entonces que la realidad depende también de lo hipertextual

Ahora, si decimos que la radio y el sonido permiten recordar un acontecimiento, es porque el sonido viaja en el tiempo pero puede perder su validez en el presente:Ong (1987) "No existe manera de detener el sonido y contenerlo. Puedo detener una cámara cinematográfica y fijar un cuadro sobre la pantalla. Si paralizo el movimiento del sonido no tengo nada: sólo el silencio, ningún sonido en absoluto", (Pag 38). Afortunadamente hoy tenemos la posibilidad de detener el sonido y capturarlo, archivarlo y reproducirlo, proceso simulado de la realidad, sin embargo esa palabra que queda es la temporalidad intacta, donde el sonido se bifurca y la memoria ya ha dejado de ser, el olvido del cual es culpable lo simulado.

Por último la palabra como hecho cultural propone la pérdida del recuerdo, lo instantáneo, de esa manera se vuelve en un hecho efímero, en la radio sucede de esta manera y no queda tiempo para el proceso interpretativo: Fernández (2003) "Pero la complejidad se hace más evidente en la medida que reconozcamos la especificidad del hecho radiofónico: toda conversación en el medio, por más que tenga una lógica interna (los interlocutores hablan y se entienden entre si) debe ser puesta en relación con el hecho de que está hecha para que otros la escuchen aunque los protagonistas lo olviden, o actúen como que olvidan" (pág. 6). Lo importante será que se pueda comprender entre la categorización de acontecimiento como un acto inacabado, esto en relación a la espectacularización del tiempo que tiene importantes relaciones en el cómo se define la forma en que el ciudadano nombra su entorno, el suceso al volverse hecho se deshace y se convierte en pérdida de memoria, tal vez un acontecimiento rompa con lo acabado y cerrado, porque la palabra radiofónica nos ubica en temporalidades, casi que es imposible reconocer un instante, sentarse a reinterpretar lo dicho.

\section{Consideraciones finales}

La radio en la cibercultura es un artefacto cultural que se sigue imaginando, sus usos van a plantearse en un mundo cada vez más sonoro pero que aún debe reivindicar la palabra como diálogo que construye, elemento importante al momento de hablar de ciudadanías.

Ya habíamos considerado de qué manera es posible que se inscriban los artefactos culturales en nuevas ciudadanías y la necesidad de que estos medios estén atentos a las temporalidades y como éstas son las que le permiten "ser ahí" en palabras de Heidegger, así es cómo también podemos estar con otros, punto para la ciudadanía, sin embargo pensar en éste fenómeno se vuelve una tarea de la complejidad debido en parte a las muchas realidades que construyen esas temporalidades, por otro lado reconocer en internet nuevas formas seguirá siendo una tarea de muchos que creen en el nuevo medio, también por eso ha sido inmanejable para los que controlan los 
mass media; todos pueden estar entonces en internet y las ciberculturas abren un camino de análisis de lo que hacemos con la web y los nuevos dispositivos, esa relación virtual nos libera y nos conecta con pensamientos más holísticos, la reivindicación de los nuevos usuarios se convierte en el punto de partida de otras realidades posibles, complejas, sistémicas, abiertas, la radio o las ciberradios se conectan con las muchas otras ciudadanías, los cambios se empiezan a gestar cada vez más rápido, el acontecimiento debe ir en contra de la velocidad implementada en la nueva sociedad que se asoma: Fernández (2003) "Por ello, el decurso de la vida social suele romper lo preconstruido introduciendo acontecimientos no previstos que ponen en cuestión esas zonas de relativa estabilidad. Para el individuo que -en su gran mayoría-no tiene posibilidades de contacto directo con ese tipo de acontecimientos, la radio es el mensajero de la novedad y, frente a él, los textos radiofónicos deberán exponer, sin demasiado enduído, los procedimientos de incorporación del acontecimiento inesperado" (Pag 6). Conectar a la radio para creer que la palabra allí habite y se quede, con nuevas y renovadas formas de recrear la trama social, construir con otros "ser ahí" y aportar para que otras ciudades sean posibles, de largo camino y de fuertes mediaciones está plagado el recorrido que empieza.

\section{Referencia Bibliográfica}

Walter J. Ong. (1987). Oralidad y escritura: tecnologías de la palabra. (México, Fondo de Cultura Económica)

Anderson, Benedict. (1993). Comunidades imaginadas: Reflexiones sobre el origen y La difusión del nacionalismo. (México, Fondo de Cultura Económica)
Levy, Pierre. (2007). Cibercultura. Informe al Consejo de Europa (Barcelona, Anthropos Editorial)

Galindo, Luis Jesús. (2005). La ciberciudad. Una visión de lo social y lo urbano desde la cibernética, la sistémica y la comunicología. (México, Andamios Año 1, número 2, junio, 2005, pp. 149-172, Universidad Autónoma de la Ciudad de México)

Baudrillard, Jean. (1978). Cultura y simulacro. (Barcelona, Editorial Kairós)

Hartog, Francois. (2003). Regímenes de historicidad.(México, Universidad Iberoamericana, Biblioteca Francisco Xavier Clavigero)

Fernandez, José Luis. (2003). El hojaldre temporal de lo radiofónico. (Figuraciones 1/2, Buenos Aires, IUNA, diciembre 2003)

Bernal Patricia. Cibercultura y ciudadanías cibernéticas: mutaciones conceptuales. http://www.ub.edu/prometheus 21/ articulos/obsciberprome/Bernal.pdf

Heidegger, Martín.(2003) El concepto de tiempo. (Madrid, Editorial Trotta)

Berttolt Brecht (1927-1932) (LaRadio: ¿Un descubrimiento antediluviano? Teórias de la radio en: Revista de Economía Política de las Tecnologías de la Información y Comunicación, www.eptic.com.br Vol.V, n.2, Mayo/Ago. 2003

Aristóteles (384-211 AC) (2000) La política. (SantaFédeBogotá,Panamericanaeditorial) 